\section{T cell depleted haploidentical transplantation: positive selection}

\section{Franco Aversa}

Pediatric Oncology/Hematology, BMT Unit, Section of Hematology, Department of Internal and Experimental Medicine, University of Perugia, Perugia, Italy

\begin{abstract}
Interest in mismatched transplantation arises from the fact that a suitable one-haplotype mismatched donor is immediately available for virtually all patients, particularly for those who urgently need an allogenic transplant. Work on one haplotype-mismatched transplants has been proceeding for over 20 years all over the world and novel transplant techniques have been developed. Some centres have focused on the conditioning regimens and post transplant immune suppression; others have concentrated on manipulating the graft which may be a megadose of extensively T celldepleted or unmanipulated progenitor cells. Excellent engraftment rates are associated with a very low incidence of acute and chronic GVHD and regimen-related mortality even in patients who are over 50 years old. Overall, event-free survival and transplant-related mortality compare favourably with reports on transplants from sources of stem cells other than the matched sibling.
\end{abstract}

\section{Introduction}

A matched sibling or a well-matched unrelated donor are the best options for patients with acute leukemia at high risk of relapse but unfortunately most patients do not have a suitable matched donor while in first hematological complete remission (CR). ${ }^{1-3}$ The only way to narrow the gap is to use a family donor with whom the patient shares one HLA haplotype for HLA-A, B, C and DR but not the other. This donor is immediately availability for all transplant candidates and unlike transplants from other alternative stem cell sources, the haploidentical transplant offers the advantage of another family member who is immediately available as an alternative donor or even a second graft from the original donor for nearly all patients who reject the graft. Furthermore any engrafted patients may potentially benefit from future attempts to modulate the cellular environment by delayed infusions of donor cell populations.
As the haplo transplant has spread to centres worldwide and several hundred patients have been treated, we have enough data to include the haploidentical option in an intention-to-treat flow-chart at least for high-risk acute leukemia patients who are the major group requiring transplantation.

\section{One-haplotype mismatched HSCT}

In this setting, our efforts have long concentrated on managing $\mathrm{T}$ cell alloreactivity in order to ensure engraftment without causing GvHD and, at the same time, minimise the major problems which are closely correlated with extensive $\mathrm{T}$ cell depletion, i.e. leukemia relapse and post transplant immune deficiency. ${ }^{4}$

In 1993, we applied the stem cell megadose in clinical practice for the first time. ${ }^{5}$ T-cell-depleted bone marrow cells were supplemented with T-cell-depleted G-CSF-mobilized peripheral blood progenitor cells (PBPCs). After the immuno-myeloablative conditioning regimen that included single fraction total body irradiation (TBI), thiotepa, cyclophosphamide (CY) and rabbit antithymocyte globulin (rATG), 36 patients received a median of $10.7 \times 10^{6} \mathrm{CD} 34^{+}$ cells/kg and $2 \times 10^{5} \mathrm{CD} 3+$ cells/kg. T-cell depletion with soybean agglutination and E-rosetting was the only prophylaxis for GvHD. A primary full-donor engraftment was achieved in $80 \%$ of patients and the incidence of acute grade II-IV GvHD was $17 \%$, in the absence of any postgrafting immunosuppression.

After this successful pilot study attention focussed on optimising graft processing and conditioning regimen. In October 1995, in an attempt to enhance immunosuppression without increasing the extra-hematologic toxicity of the conditioning, we substituted fludarabine for $\mathrm{CY}$ in the pretransplant chemotherapy.6,7 Our adult patients receive $8 \mathrm{~Gy}$ TBI in a single fraction at high dose rate with lung shielding. To prevent growth inhibition TBI is fractionated in children. The total dose is 12 Gy over 3 days. Since we introduced these two modifications - fludarabine and 4 Gy TBI to the lung we have had no cases of idiopathic interstitial pneumonitis. As far as regards ATG, we adjusted our doses according to the source (6-10 $\mathrm{mg} / \mathrm{kg}$ thymoglubulin, 20-25 mg/kg Fresenius, all over 5 days) (Figure 1). Furthermore, with the aim of eliminating GvHD we tried to reduce the number of $\mathrm{T}$ lymphocytes in the inoculum to a mean of $2 \times 10^{4} / \mathrm{kg}$, i.e. one log less than in the previous series of patients. The technical inconveniences of the our original graft processing procedure were overcome when, in January 1999, we started positively selecting the CD34+ cells using the Clinimacs device which in a one-step, fully automated procedure selects a highly purified CD34+ cell population. Our final inocula now contains a median of $12 \times 10^{6} \mathrm{CD} 34^{+}$cells $/ \mathrm{kg}$ and $1 \times 10^{4}$
Correspondence: Franco Aversa, Pediatric Oncology/ Hematology, BMT Unit, Section of Hematology, Department of Internal and Experimental Medicine, University of Perugia, Perugia, Italy.

E-mail: aversa@unipg.it

Key words: T cell depleted haploidentical transplantation.

Received for publication: 4 May 2011.

Accepted for publication: 4 June 2011.

This work is licensed under a Creative Commons Attribution NonCommercial 3.0 License (CC BYNC 3.0).

(C) Copyright F. Aversa, 2011

Licensee PAGEPress, Italy

Pediatric Reports 2011; 3(s2):e14

doi:10.4081/pr.2011.s2.e14

CD3+ cells/kg recipient body weight. Finally, given its immunosuppressive properties, we also suspended post-transplant G-CSF administration to the recipients (Table 1). ${ }^{8,9}$

\section{Results}

The extra-hematological toxicity of this innovative conditioning regimen was minimal even in patients who were at risk because $25 \%$ were in the upper age group for transplantation, between 45 and 68 years, and about $50 \%$ were in advanced stage disease.

Under protocols that include fludarabine we have achieved a $95 \%$ primary engraftment rate. Acute and chronic GvHD were almost completely prevented with ex vivo positive selection of the grafts and in vivo $\mathrm{T}$ cell depletion exerted by ATG.

With minimal GvHD one might expect a high relapse rate in patients who were at high risk of relapse at transplant and did not benefit from the GvHD-related GvL effect. However, the relapse rate was $17 \%$ in AML and $27 \%$ in ALL patients who were transplanted in any CR (Figure 2a). Our intensely myeloablative conditioning regimen might have compensated for the lack of T-cell mediated GvL effect. Furthermore, unlike matched transplants, haploidentical transplants can benefit from a NKcell mediated GVL effect. ${ }^{10-13}$ Indeed, patients transplanted in remission had a $3 \%$ and $47 \%$ probability of relapse respectively whether the donor was NK alloreactive or not (Figure $2 b$ ). Therefore, given this evidence, donor-vs-recipient NK alloreactivity, as predicted by the HLA disparity, should be exploited when selecting the optimal donor from among the mismatched family members. Cytomegalovirus (CMV) was the main cause of infection-related deaths in 


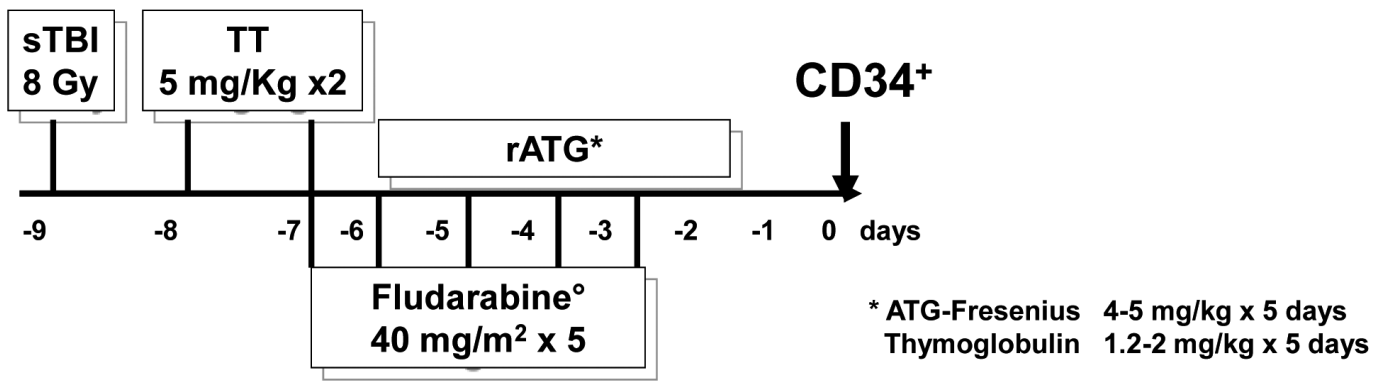

${ }^{\circ} \mathrm{CY}$ in the first 36 patients $(<1995)$

\begin{tabular}{|l|l|} 
Lung shielding: & $\begin{array}{l}\text { TBI is given at } 2 \mathrm{~Gy} \times 2 \times 3 \text { days } \\
\text { (total dose } 12 \mathrm{~Gy} \text {, lungs } 9 \mathrm{~Gy} \text { ) } \\
\text { in recipients } \leq 12 \text { yrs of age }\end{array}$ \\
\hline $4 \mathrm{~Gy} 1995 \rightarrow$ &
\end{tabular}

Figure 1. Conditioning regimen.

our patients. Other infections which contribute to our $40 \%$ incidence of non relapse mortality include fungi and bacteria (Figure 3a). Our anti-infective approach is mainly based upon ganciclovir over the conditioning followed by foscarnet for the first three weeks after transplanation. Liposomal amphotericine $B$ is the standard anti-Aspergillus propylaxis until the neutrophil count normalises. Overall, disease status at transplant is a significant factor for TRM and the risk of life threatening infections plateaus out after about one year when the immunity tends to normalize. On the other hand we have less mortality in children and adolescents, particularly when they are transplanted in remission (Figure $3 \mathrm{~b}$ ).

Our overall population of 284 patients has a maximum follow-up now of 17 years. DFS ranged from $30 \%$ in ALL to $43 \%$ in AML in patients who were transplanted in any CR and it is worth nothing that, with no chronic GvHD, all these long term survivors have an excellent quality of life. We have $60 \%$ survival in patients aged 18 or under. As might be expected, outcomes are extremely poor in patients who are transplanted in chemoresistant relapse. However our 18\% DFS for advanced AML patients is really quite good, considering that they had no other option beyond the haplo-transplant (Figure 4). Having a NK alloreactive donor im-

Table 1. Modifications in the Perugia Haplotransplant Programme.

\begin{tabular}{|c|c|c|c|}
\hline Years of study & 1993-1995 & 1995-1998 & $1999-2008$ \\
\hline n. of patients & 36 & 44 & 196 \\
\hline $\begin{array}{l}\text { Age (y) } \\
\text { Median (range) }\end{array}$ & $25(2-51)$ & $24(4-53)$ & $34(6-68)$ \\
\hline HSC source & $\mathrm{BM}+\mathrm{PB}$ & $\begin{array}{l}\text { BM+PB in } 29 \\
\text { PB only in } 15\end{array}$ & $\mathrm{~PB}$ \\
\hline Graft processing & $\mathrm{SBA}+\mathrm{E}$ rosette & $\begin{array}{l}\text { E rosette+ } \\
\text { CD34+ selection } \\
\text { (Ceprate SC) }\end{array}$ & $\begin{array}{l}\text { CD34+ selection } \\
\text { (Clinimacs) }\end{array}$ \\
\hline Conditioning regimen & TBI, TT, CY, ATG & TBI, TT, F, ATG & TBI, TT, F, ATG \\
\hline G-CSF posttransplant & YES & YES & NO \\
\hline $\begin{array}{l}\text { Graft content, median } \\
\text { CD34+/kg } \\
\text { CD3+/kg }\end{array}$ & $\begin{array}{l}10.8 \times 10^{6} \\
2.2 \times 10^{5}\end{array}$ & $\begin{array}{c}10.5 \times 10^{6} \\
0.2 \times 10^{5}\end{array}$ & $\begin{array}{c}12.8 \times 10^{6} \\
0.1 \times 10^{5}\end{array}$ \\
\hline $\begin{array}{l}\text { Diseases } \\
\text { AML / ALL }\end{array}$ & $12 / 24$ & $21 / 23$ & $131 / 65$ \\
\hline $\begin{array}{l}\text { Disease status at TX } \\
\text { CR } 1 \\
\text { CR } \geq 2 \\
\text { Relapse }\end{array}$ & $\begin{array}{c}0 \\
18 \\
18\end{array}$ & $\begin{array}{c}7 \\
22 \\
15\end{array}$ & $\begin{array}{l}63 \\
64 \\
69\end{array}$ \\
\hline
\end{tabular}

HSC: Hematopoietic Stem Cells; BM: Bone Marrow; PB: Peripheral Blood; SBA: Soybean Agglutinin; TBI: Total Body Irradiation; TT: Thiotepa; CY: Cyclophosphamide; ATG: Anti Thymocyte Globulin; G-CSF: Granulocyte Colony Stimulating Factor; AML: Acute Myeloid Leukemia; ALL: Acute Lymphoid Leukemia; CR: Complete Remission. 

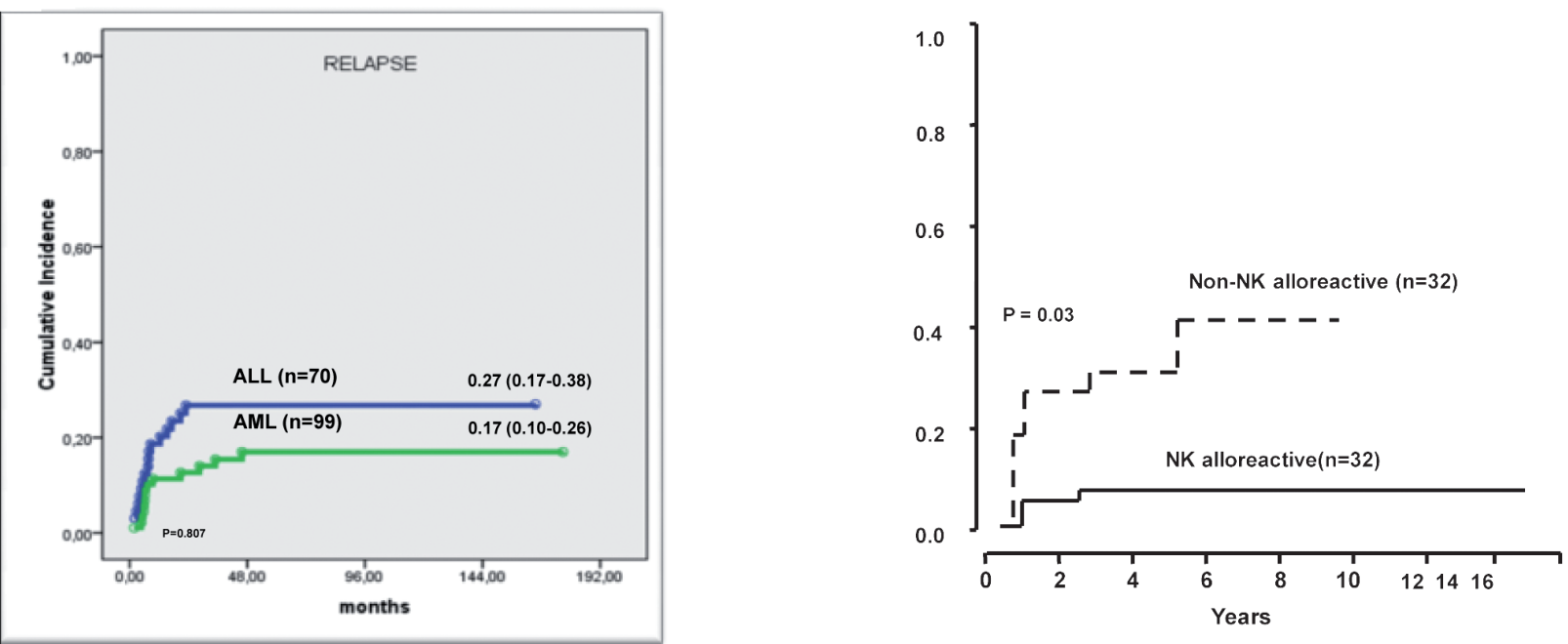

Figure 2. Leukemia relapse.

A) All patients $(n=284)$

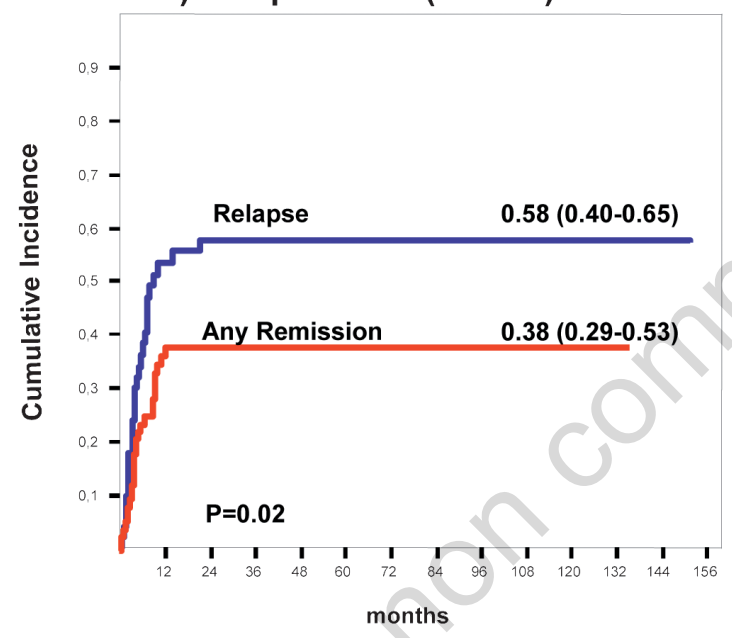

B) Patients aged $\leq 18$ yrs $(n=68)$

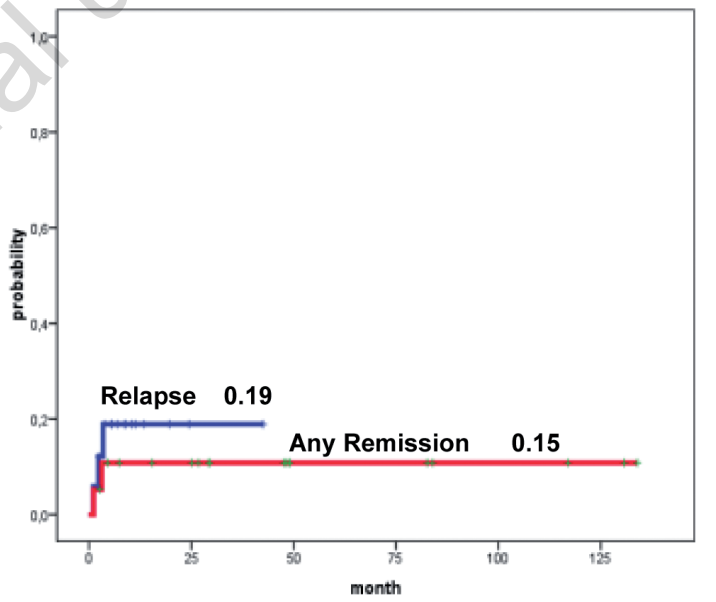

Figure 3. Non-relapse mortality.

pacts strongly on outcomes in patients with AML in remission. 13

Another positive factor which emerged from Martin Stern's analysis was that a maternal donor is associated with best survival. In a retrospective analysis of 118 patients, having a mother who can exert donor vs recipient NK cell alloreactivity offers an excellent probability of survival. 14

In conclusion, the ideal haplotransplant should be structured as follows: patients should be in CR, the donor should be selected according to CMV serology and should perferably be a mother and /or someone who can exert donor vs recipient NK cell alloreactiviy. TBI can be given as a single or fractionated schedule. Lung shielding is important. Fludarabine is better than $\mathrm{CY}$ and the recommended ATG doses are $25 \mathrm{mg} / \mathrm{kg}$ for Fresenius and $6 \mathrm{mg} / \mathrm{kg}$ for thymoglobulin. Extensive T and $\mathrm{B}$ cell depletion is crucial for success and of course a megadose of CD34+ cells must be reached. Post-transplant the only recommendation is for anti-infective prophylaxis.
The present challenge is to reduce posttransplant infectious mortality in adults and researchers are focussing on strategies to ensure faster and better immunological reconstitution separating GvHD from favourable donor immune responses.

In extensively $\mathrm{T}$ cell depleted transplants such as we use in Perugia, strategies are based on post-transplant addbacks of donor $\mathrm{T}$ cells.

To prevent GvHD, these addbacks are purged of alloreactivity using a photodynamic method. ${ }^{15}$ 

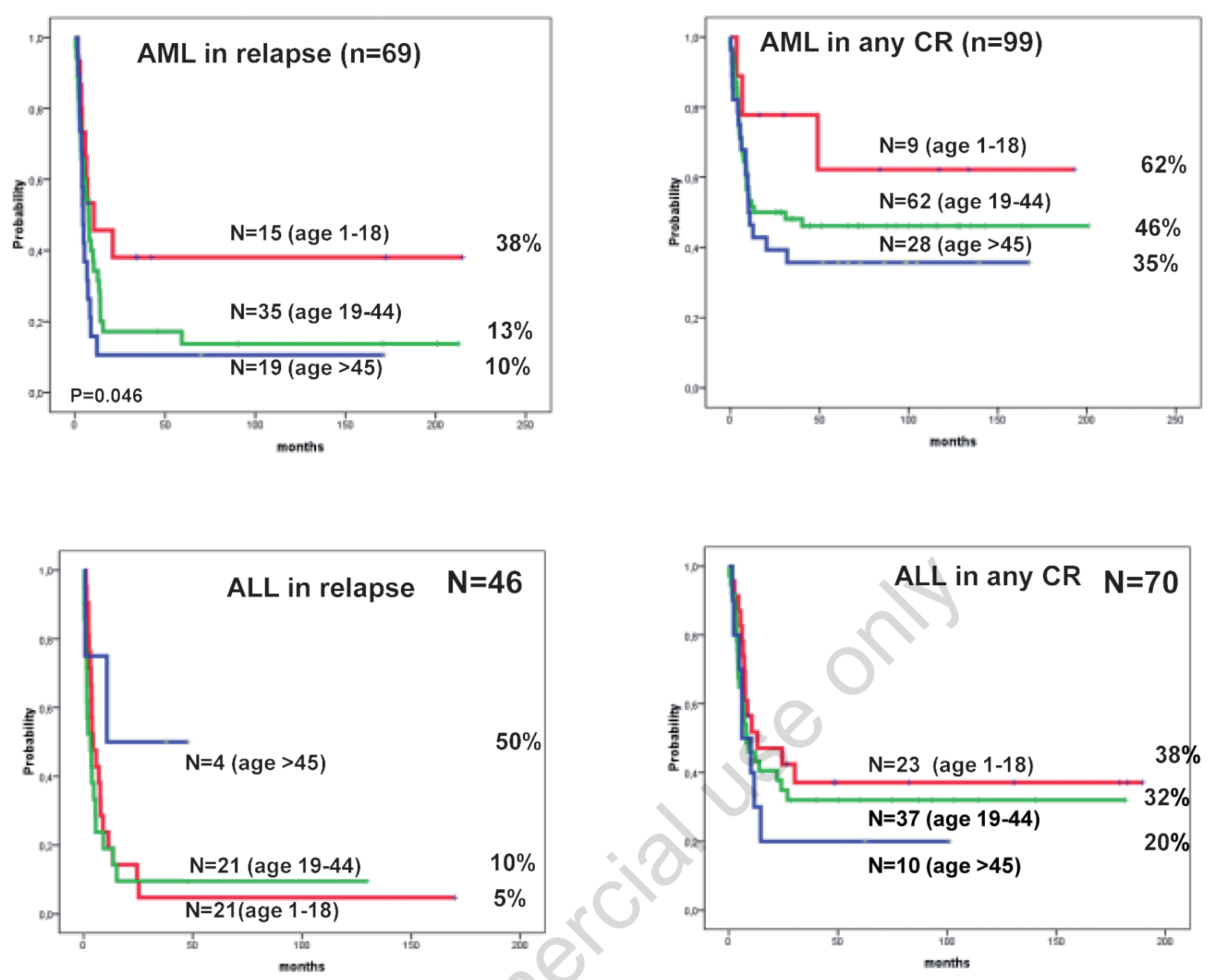

Figure 4. Disease-free survival.

We are also attempting to down modulate alloreactivity by infusion of naturaly occurring donor T regulatory cells. ${ }^{16}$

In conclusion, today we are in a position to offer a transplant to all patients with high-risk leukaemia. HLA typing must be done at diagnosis. If a sibling donor is not available attention should focus on alternative sources of stem cells limiting the registry search for a well-matched unrelated donor to 2 or 3 months. If unsuccessful, consider a cord blood unit or a haplo donor. Both are immediately available but an haploidentical NK alloreactive donor offers a better outcome and survival.

\section{References}

1. Gupta V. Alternative donor transplants for high-risk acute myeloid leukemia. Curr Opin Hematol 2008;15:115-20.
2. Bachanova V, Weisdorf D. Unrelated donor allogeneic transplantation for adult acute lymphoblastic leukemia: a review. Bone Marrow Transplant 2008;41:455-64.

3. Rocha V, Gluckman E. Eurocord-Netcord registry and European Blood and Marrow Transplant group. Improving outcomes of cord blood transplantation: HLA matching, cell dose and other graft- and transplantation-related factors. Br J Haematol 2009; 147:262-74.

4. Reisner Y, Martelli MF. Stem cell escalation enables HLA-disparate haematopoietic transplants in leukaemia patients. Immunol Today 1999;20:343-7.

5. Aversa F, Tabilio A, Terenzi A, et al. Successful engraftment of T-cell-depleted haploidentical "three-loci" incompatible transplants in leukemia patients by addition of recombinant human granulocyte colony-stimulating factor-mobilized peripheral blood progenitor cells to bone marrow inoculum. Blood 1994;84:3948-55.

6. Terenzi A, Aristei C, Aversa F, et al. Efficacy of fludarabine as an immunosuppressor for bone marrow transplantation conditioning: Preliminary results. Transplant Proc 1996;28:3101.

7. Aversa F, Tabilio A, Velardi A, et al. Treatment of high risk acute leukemia with Tcell-depleted stem cells from related donors with one fully mismatched HLA haplotype. N Engl J Med 1998;339:1186-93.

8. Aversa F, Terenzi A, Tabilio A, et al. Fullhaplotype mismatched hematopoietic stem cell transplantation: A phase II study in patients with acute leukemia at high risk or relapse. J Clin Oncol 2005;23:3447-54.

9. Volpi I, Perruccio K, Tosti A, et al. Postgrafting granulocyte colony-stimulating factor administration impairs functional immune recovery in recipients of HLA haplotype-mismatched hematopoietic transplants. Blood 2001;97:2514-21. 
10. Ruggeri L, Capanni M, Casucci M, et al. Role of natural killer cell alloreactivity in HLA-mismatched hematopoietic stem cell transplantation. Blood 1999;94:333-9.

11. Velardi A, Ruggeri L, Moretta A, Moretta L. NK cells: a lesson from mismatched hematopoietic transplantation. Trends Immunol 2002;23:438-44.

12. Ruggeri L, Capanni M, Urbani E, et al. Effectiveness of donor natural killer cell alloreactivity in mismatched hematopoietic transplants. Science 2002;295:2097-2100. 13. Ruggeri L, Mancusi A, Capanni M, et al. Donor natural killer cell allorecognition of missing self in haploidentical hematopoietic transplantation for acute myeloid leukemia: challenging its predictive value. Blood 2007;110:433-40.

14. Stern M, Ruggeri L, Mancusi A, et al. Survival after T cell-depleted haploidentical stem cell transplantation is improved using the mother as donor. Blood 2008;112:2990-5.
15. Perruccio K, Topini F, Tosti A, et al. Photodynamic purging of alloreactive T cells for adoptive immunotherapy after haploidentical stem cell transplantation. Blood Cells Mol Dis 2008;40:76-83.

16. Di Ianni M, Falzetti F, Carotti A, et al. Adoptive Immunotherapy with Tregs Prevents GvHD and Favours Immune Reconstitution After HLA Haploidentical Transplants for Hematological Malignancies. Blood (ASH Annual Meeting Abstracts) 2009;114:Abstract 4. 